\title{
Phase transitions in ferro-antiferromagnetic bilayers with a stepped interface
}

\author{
D.P. Landau ${ }^{\text {a,* }}$, Shan-Ho Tsai ${ }^{\text {a,c }}$, Thomas C. Schulthess ${ }^{\text {b }}$ \\ ${ }^{a}$ Center for Simulational Physics, University of Georgia, Athens, GA 30602 \\ ${ }^{\mathrm{b}}$ Center for Computational Sciences and Computer Science \& Mathematics Division, Oak Ridge National Laboratory, \\ Oak Ridge, TN 37831 \\ ${ }^{\mathrm{c}}$ Enterprise IT Services, University of Georgia, Athens, GA 30602
}

\begin{abstract}
We have studied magnetic ordering in ferro/antiferromagnetic (F/AF) bilayers using Monte Carlo simulations of classical Heisenberg spins. For both flat and stepped interfaces we observed order in the AF above the Néel temperature, with the AF spins aligning collinearly with the F moments. In the case of the stepped interface there is a transition from collinear to perpendicular alignment of the $\mathrm{F}$ and $\mathrm{AF}$ spins at a lower temperature.
\end{abstract}

Key words: Ferromagnetic-antiferromagnetic bilayer, classical spin, anisotropy, Monte Carlo simulation PACS: $75.70 .-\mathrm{i}, 75.10 . \mathrm{Hk}, 75.40 . \mathrm{Mg}$

Magnetic properties of ferro/antiferromagnetic (F/AF) bilayers can be very different from those of free $\mathrm{F}$ and $\mathrm{AF}$ films. These coupled systems exhibit a shift in the hysteresis loop (exchange bias) and larger coercivity than the free $\mathrm{F}$ film [1]. Other effects of the $\mathrm{F} / \mathrm{AF}$ coupling include the order in the AF observed above the Néel temperature[2] and the perpendicular orientation of the $\mathrm{F}$ with the AF moments[3,4]. Understanding these effects and the nature of the $\mathrm{F} / \mathrm{AF}$ interfaces remains a challenge.

In this paper we use Monte Carlo simulations to study the effect of the interfacial exchange and roughness on the magnetic ordering in F/AF bilayers. The work is motivated by recent experiments for $\mathrm{Fe}_{3} \mathrm{O}_{4} / \mathrm{CoO}$ multilayers[2]. We consider a ferromagnetic (F) film coupled to an antiferromagnetic (AF) film where the lattice is coherent across the $\mathrm{F} / \mathrm{AF}$ interface. Each film is a bcc lattice, with linear sizes $L_{x}=L_{y}=L \leq 96$ and 12 staggered (be-

\footnotetext{
* Corresponding author. Tel +1 (706) 542 2908, Fax +1 (706) 5422492

Email address: dlandau@uga.edu (D.P. Landau).
}

cause of the bcc structure) layers of classical spins $\left|\mathbf{S}_{\mathbf{r}}\right|=1$, which interact via the Hamiltonian

$$
\begin{gathered}
\mathcal{H}=-J_{F} \sum_{\left\langle\mathbf{r}, \mathbf{r}^{\prime}\right\rangle \in \mathrm{F}} \mathbf{S}_{\mathbf{r}} \cdot \mathbf{S}_{\mathbf{r}^{\prime}}-K_{F} \sum_{\mathbf{r} \in \mathrm{F}}\left(S_{\mathbf{r}}^{z}\right)^{2}- \\
-J_{A} \sum_{\left\langle\mathbf{r}, \mathbf{r}^{\prime}\right\rangle \in \mathrm{AF}} \mathbf{S}_{\mathbf{r}} \cdot \mathbf{S}_{\mathbf{r}^{\prime}}-K_{A} \sum_{\mathbf{r} \in \mathrm{AF}}\left(S_{\mathbf{r}}^{y}\right)^{2}-\underset{\left\langle\mathbf{r}, \mathbf{r}^{\prime}\right\rangle \in \mathrm{F} / \mathrm{AF}}{J_{I}} \sum_{\mathbf{r}} \cdot \mathbf{S}_{\mathbf{r}^{\prime}}
\end{gathered}
$$

where $\left\langle\mathbf{r}, \mathbf{r}^{\prime}\right\rangle$ denotes nearest-neighbor pairs of spins coupled with exchange interactions $J_{F}=$ $5 J>0$ in the $\mathrm{F}$ film, $J_{A}=-J<0$ in the $\mathrm{AF}$ film, and $J_{I}=-J$ at the $\mathrm{F} / \mathrm{AF}$ interface. Spins in the AF film have a uniaxial single-site anisotropy $K_{A}=J$, whose easy axis is along the $y$ axis. The demagnetizing field on the F film is modeled with a hard-axis $\left(K_{F}=-0.5 J\right)$ along the $z$ direction, which is perpendicular to the $\mathrm{F} / \mathrm{AF}$ interfacial plane. No external magnetic field is applied. We use periodic boundary conditions along the $x$ and $y$ directions and free boundary conditions along the $z$ direction. We model flat interfaces that are fully uncompensated as well as uniformily stepped ones that are compensated on average. 
The stepped interface has $6, L$, and one spin per terrace in the $x, y$, and $z$ directions, respectively.

The $\mathrm{F}$ and $\mathrm{AF}$ order parameters are the uniform $(m)$ and staggered $\left(m_{s}\right)$ magnetization per spin, respectively[6]. $m_{x}$ and $m_{y}$ denote the two components of $m$ in the interfacial plane. We perform Monte Carlo simulations with Metropolis algorithm at fixed temperature $T$. Typically we discard $3 \times 10^{5}$ Monte Carlo Steps/site (MCS) for thermalization and then use about $2 \times 10^{5}$ MCS for averages. Whenever not shown, error bars in the figures are smaller than the symbol sizes.

The $T$-dependence of $m$ shown in Figs.1(a) and 1(b) plus finite-size analysis (not shown) indicate an $\mathrm{F}$ ordering transition at $T_{c} \approx 9.3 J / k_{B}$. A free 12-atomic-layer AF film undergoes a phase transition at the Néel temperature[5] $T_{N} \approx 2.2 J / k_{B}$. For

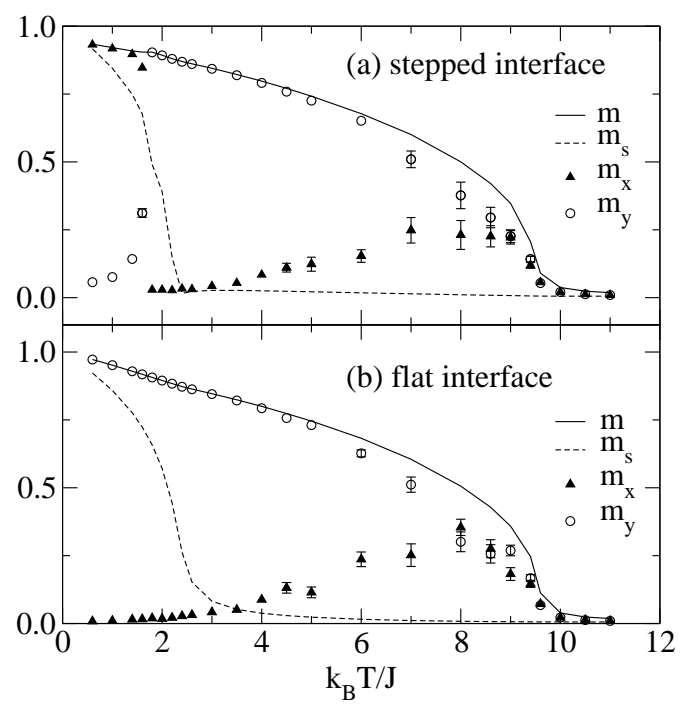

Fig. 1. Temperature dependence of $m, m_{s}, m_{x}$, and $m_{y}$ (see text) for $L=60$, with (a) stepped and (b) flat interface.

both flat and stepped interfaces, the spins on the $\mathrm{F}$ film orient predominantly in a direction collinear with the easy axis of the AF at high $T$ (see Fig.1), even above $T_{N}$. This is an indication that there is still order in the AF above $T_{N}$ due to the coupling to the ferromagnet. As $T$ is lowered in the case of the stepped interface the $\mathrm{F}$ spins switch to orient in a direction that is perpendicular to the AF spins. Our results suggest that the onset of this perpendicular orientation is very sharp and it occurs at a temperature below $T_{N}$. The $z$-components of $m$ and $m_{s}$ are very small for all $T$. In the absence of the AF film, spins on the F film have global rotation symmetry in the $x$ - $y$ plane, which is their easy plane. The preferential orientations of the F spins observed either below or above $T_{N}$ result from the exchange coupling to the AF film.

Fig.2 shows $m_{s}$ versus $T$ for different $L$ for stepped and flat interfaces. In the former case the decay to zero of $m_{s}$ at $T \approx 2.2 J / k_{B}$ becomes sharper for larger $L$ (see the inset), suggesting an $\mathrm{AF}$ phase transition at $T_{N}$. In contrast, with a flat (uncompensated) interface there is no finite-size dependency of $m_{s}$ near $T_{N}$, suggesting the absence of an $\mathrm{AF}$ phase transition there.

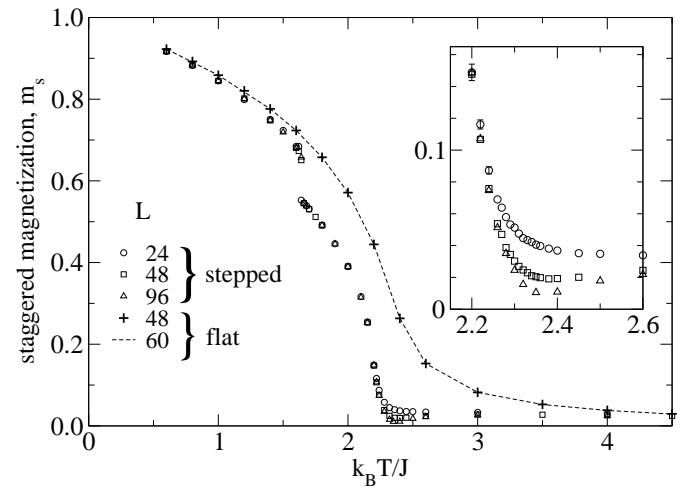

Fig. 2. Staggered magnetization as a function of temperature for stepped and flat interfaces.

Research partially supported by NSF grant DMR-0094422 and DOE-OS/ASCR through the Laboratory Technology Research Program under contract DE-AC05-00OR22725 with UT-Battelle, LLC. Simulations performed on the IBM SP at NERSC and ORNL.

\section{References}

[1] See e.g., J. Nogués and I.K. Schuller, J. Magn. Magn. Mater. 192 (1999) 203; A.E. Berkowitz and K. Takano, ibid. 200 (1999) 552; M. Kiwi, ibid. 234 (2001) 584; R.L. Stamps, J. Phys. D 33 (2000) R247.

[2] P.J. van der Zaag, Y. Ijiri, J.A. Borchers, L.F. Feiner, R.M. Wolf, J.M. Gaines, R.W. Erwin, and M.A. Verheijen, Phys. Rev. Lett. 84 (2000) 6102.

[3] N.C. Koon, Phys. Rev. Lett. 78 (1997) 4865; T.C. Schulthess and W.H. Butler, ibid. 81 (1998) 4516.

[4] T.J. Moran and I.K. Schuller, J. Appl. Phys. 79 (1996) 5109; Y. Ijiri, J.A. Borchers, R.W. Erwin, S.-H. Lee, P.J. van der Zaag, and R.M. Wolf, Phys. Rev. Lett. 80 (1998) 608; T.J. Moran, J. Nogués, D. Lederman, and I.K. Schuller, Appl. Phys. Lett. 72 (1998) 617.

[5] S.-H. Tsai, D.P. Landau, T.C. Schulthess, J. Appl. Phys. 91 (2002) 6884.

[6] S.-H. Tsai, D.P. Landau, T.C. Schulthess, J. Appl. Phys. 93 (2003) 8612. 